
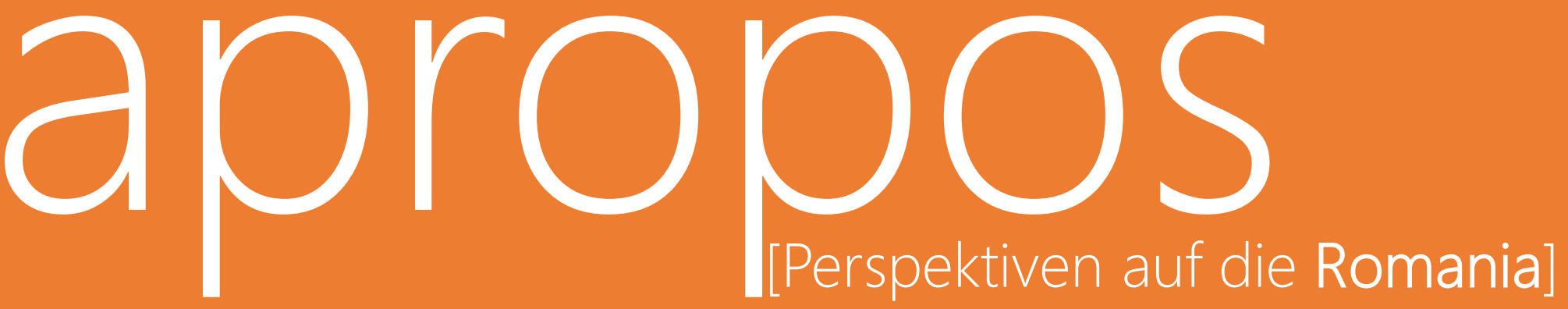

Sprache/Literatur/Kultur/Geschichte/Ideen/Politik/Gesellschaft

Paul Claudel et la modernité médiévale

Penser, écrire et réécrire le Moyen Âge claudélien

Jean-François Poisson-Gueffier

apropos [Perspektiven auf die Romania] hosted by Hamburg University Press

2021, 6

pp. $200-211$

ISSN: 2627-3446

Online

https://journals.sub.uni-hamburg.de/apropos/article/view/1705

Zitierweise

Poisson-Gueffier, Jean-François . 2021. „Paul Claudel et la modernité médiévale. Penser, écrire et réécrire le Moyen Âge claudélien", apropos [Perspektiven auf die Romania] 6/2021, 200-211. doi: 10.15460/apropos.6.1705 


\title{
Jean-François Poisson-Gueffier
}

\section{Paul Claudel et la modernité médiévale \\ Penser, écrire et réécrire le Moyen Âge claudélien}

\author{
Jean-François Poisson-Gueffier \\ est professeur agrégé de lettres au \\ Lycée Pierre d'Ailly, Compiègne. \\ jeanfran_2@yahoo.fr
}

Mots-clés

Paul Claudel - Moyen Âge - médiévalisme - médiévité - modernité

\section{Considérations introductives}

Cet article présente la méthode et les principales conclusions d'un ouvrage intitulé Paul Claudel et le Moyen Âge (Poisson-Gueffier 2022), à paraitre en mars 2022 chez Honoré Champion, dans la collection "Poétiques et Esthétiques XXe-XX|e siècles ». Son écriture procède d'une béance dans la littérature critique consacrée à l'œuvre du Poëte. Si les versants extrême-orientaux et exégétiques bénéficient de très nombreuses études qui éclairent les fondements les plus intimes de sa foi et documentent une part déterminante de sa carrière diplomatique, le Moyen Âge n'a été abordé que de manière latérale, sans ambition de penser comme une totalité les allusions et réalités médiévales présentes dans son œuvre. Paradoxalement, seul un article cursif de Jacques Madaule (Madaule 1990, p. 167-188) aborde de front le Moyen Âge claudélien, dont la richesse mérite une tout autre considération.

Qu'il en célèbre les figures ou s'approprie les éléments de la pensée et de la sensibilité médiévales, Claudel intègre dans la matière de son Journal, de ses dialogues, de ses poèmes et de ses drames, les traces d'une temporalité qui demeure présente et revivifiée. Dans cette perspective, il devrait bénéficier d'une attention particulière, dans le cadre du médiévalisme, mouvement dont l'ambition revient à étudier la « référence discursive au Moyen Âge » (Grévin 2015) ${ }^{1}$.

L'œuvre de Claudel n'en est pas moins rejetée dans l'ombre de ce mouvement. Alors que la « médiévité » (selon le néologisme de Michèle Gally, v. Gally 2000) de

\footnotetext{
${ }^{1}$ Voir également les travaux de Francis G. Gentry et Ulrich Müller qui ont déterminé quatre modèles de réception du Moyen Âge : la refonte originale de thèmes sujets, œuvres et thèmes médiévaux; la reconstitution " authentique "; la réception académique ; la réception politique et idéologique (Gentry et Müller 1991, p. 401).
} 
Rostand (La Princesse lointaine), Proust, Gracq (Le Roi Pêcheur) ou Jacques Roubaud (Graal-Théâtre, Le Grand incendie de Londres) a été largement étudiée, celle de Claudel demeure largement ignorée. La représentation du Poëte par ses contemporains donne sans doute l'une des clés de ce renoncement. Ainsi, les détracteurs de Paul Claudel n'ont cessé de le dépeindre comme un " homme du Moyen Âge » (Gregh 1955, p. 579), qui « ne voit de salut que dans le haut Moyen Âge chrétien, vers lequel il voudrait nous faire remonter » (Poizat 1927, p. 34). L'inspiration médiévale de Claudel dénierait ainsi à son art toute modernité. Considéré comme un homme médiéval, il incarnerait un monde révolu, à une époque où le progrès est un concept majeur.

Alors que d'autres auteurs incluraient le Moyen Âge dans leur œuvre de manière pleinement réflexive, consciente de l'écart entre deux temporalités distinctes dont le hiatus représente un intérêt littéraire de premier ordre, Claudel n'est ainsi considéré que comme un "homme du Moyen Âge ». Non comme un artiste qui puise aux sources de l'imaginaire et des lettres médiévales la matière de l'œuvre à venir, mais comme un auteur inscrit dans une autre temporalité, au point d'en négliger la sienne. Ce raisonnement, empreint de malveillance, procède moins d'une lecture véritable que d'une reductio ad absurdum. La notion d'écart esthétique semble ainsi rejetée d'un revers de main. L'approche de la médiévité claudélienne doit être abordée avec nuance et sans a priori, pour restituer les linéaments de son projet et la valeur intrinsèque de son œuvre, qui ne se recouvrent pas entièrement. La civilisation médiévale - concept que nous préservons, malgré ses limites (cf. Stahuljak 2020) - est subordonnée, dans la perspective du Poëte, à une volonté d'édification. La dimension chrétienne et pastorale du théâtre claudélien est manifeste et, à cet égard, le Moyen Âge correspond à un âge d'or, durant lequel la pensée, la société et le pouvoir politique étaient dominés par l'influence ecclésiale.

Ce projet dramatique et poétique d'édification et d'exaltation de la religion repose sur l'accueil dans son œuvre de toutes les réalités du monde sous le signe de la Croix, et donne précisément lieu à un malentendu fondamental, voire plus souvent encore à une défiguration cynique de son message. Accueillir le Moyen Âge induirait de décalquer des formes révolues, de se tourner vers une antériorité qui lesterait sa parole et sa pensée de considérations doctrinales détachées de l'esprit matérialiste et positiviste qui marque la jonction du XIX et du XX ${ }^{e}$ siècle. Certes, la médiévité claudélienne semble d'aspect plus austère que le Moyen Âge de fantaisie et de merveille, de barbarie et d'onirisme privilégié dans d'autres media. Le projet claudélien de célébration du monde a quelque peu éclipsé une œuvre dont la lecture montre la profusion et la richesse. Loin d'inscrire ses pas dans ceux de modèles médiévaux, Claudel repense, infléchit, hybride une matière d'une remarquable ductilité.

Si les clercs médiévaux fondaient leurs écrits sur l'auctoritas et concevaient la Bible comme point d'origine absolu, Claudel ajoute à cette perspective la médiation de la théologie, de l'histoire et de la littérature médiévales. Ce qui recouvre en partie ce qu'Alain Corbellari définit comme une nouvelle modalité de lecture typologique de la Bible, "une sorte de cinquième sens de l'Écriture», "en clé nationale 
française » (Corbellari 2008, p. 286). Le Moyen Âge claudélien retire de son hybridité une part fondamentale de sa modernité. II n'est jamais envisagé comme un cadre absolu, mais toujours en corrélation avec d'autres temps (en-deçà et audelà du Moyen Âge) et d'autres lieux (la France et l'Europe s'ouvrent à des imaginaires empruntés au monde entier).

Le malentendu ainsi décelé trouve un élément de résolution. La perspective catholique adoptée par Claudel n'a pas pour ambition d'être moderne mais le devient à travers le travail de l'artiste - dénomination à laquelle tient le Poëte. En ce sens, célébrer le monde revient à inclure les éléments du monde, moins à les fusionner tout à fait qu'à les fondre en partie dans une matière qui en préserve les aspérités. L'écart esthétique, que lui dénient certains de ses contemporains, est alors manifeste : la médiévité claudélienne est d'une grande richesse parce qu'elle multiplie les consonances et les dissonances avec d'autres matières, d'autres lieux et d'autres siècles.

En vue de donner un aperçu de cette hybridation, il convient de préciser la diversité des genres et des formes auxquels empruntent les principales pièces du corpus. L'univers dramatique se fonde parfois sur une dominante médiévale, mais le plus souvent, le Moyen Âge n'est que la composante d'un système complexe. Dans L'Homme et son désir se conjuguent des échos de la moralité médiévale, du drame amazonien et du conte chinois, Jeanne d'Arc au Bûcher s'inspire du théâtre nô et du drame liturgique (Chujo 1990), La Danse des Morts du drame liturgique et du dithyrambe grec, le Livre de Christophe Colomb de la matière antique, du nô, de la liturgie catholique et de l'épicisation au sens brechtien (cf. Sarrazac 2010, p. 74-75).

L'écriture de Paul Claudel et le Moyen Âge est ainsi dominée par une double ambition apparemment contradictoire et qui, s'agissant d'un pan négligé de son $œ u v r e$, reflète à la fois un esprit d'aventure et de prudence. L'œuvre de Claudel étant dense et complexe, l'essai présente une synthèse des formes que revêt un Moyen Âge multiple. Au-delà de ce premier état des lieux, qui invite à une réflexion historique, anthropologique, culturelle et littéraire, se révèlent les virtualités du médiévalisme claudélien - formulons le vœu que ces pages posent les fondements de futures études qui lui rendent pleinement justice. Une œuvre aussi vaste et plurielle rend nécessairement incomplète l'élaboration d'une synthèse, tant sont nombreuses les questions et problématiques dont une notice ne saurait épuiser le sens.

Afin de tendre vers une certaine forme de cohérence, nous avons accordé une importance fondamentale à quelques œuvres emblématiques des ressources du Moyen Âge claudélien. Ce « corpus médiéval » constitue un principe et non une fin : La Jeune fille Violaine, L'Annonce faite à Marie, L'Homme et son désir, Jeanne d'Arc au Bûcher, Le Livre de Christophe Colomb et La Danse des Morts en sont les premiers représentants. Plus largement, nous avons considéré l'intégralité de I'œuvre dramatique, le Journal, l'CEuvre poétique et les CEuvres en prose, soit I'ensemble des volumes parus en Pléiade. Dans une approche ouverte et nondogmatique, nous avons intégré tout ce qui relevait, en quelque manière, du Moyen Âge. Naturellement, le texte claudélien s'est doublé d'une littérature 
critique qui n'a pas manqué d'aborder de manière ponctuelle ces questions médiévales, parfois embrassées en un geste plus large ${ }^{2}$.

Paul Claudel et le Moyen Âge a pour singularité d'étudier de manière systématique l'ensemble que constitue ce système de références médiévales, et plus encore d'inverser le regard porté sur l'œuvre. À un regard de claudélien le cède un regard de médiéviste, de telle sorte que l'on ne part plus du XXe siècle pour analyser les rémanences du Moyen Âge, mais du Moyen Âge pour analyser une œuvre du XXe siècle. Cette translation du regard invite à restituer à cette œuvre sa part esthétique et expérimentale. Réciproquement, ce que les études médiévales peuvent apporter aux études claudéliennes trouve un équivalent dans ce que les études claudéliennes peuvent apporter à la théorie - toujours en devenir - du médiévalisme.

Le Moyen Âge de Claudel n'est en rien un Moyen Âge « de convention » (L'Annonce faite à Marie, 2011, p. 991) et n'implique en rien une régression. Il est, à sa manière, infiniment plus moderne que celui de ses contemporains. Ainsi, le Mystère claudélien tire bénéfice de ses réflexions sur l'espace scénique et creuse un écart notable avec les Mystères médiévaux, dont il repense en profondeur la forme et le sens.

Cette matière est présentée dans un parcours en trois temps. Le premier, "Penser », ordonne sa vision particulière du Moyen Âge, afin de déterminer ce que représentent pour lui ces dix siècles. Les deux premières parties abordent successivement le monde médiéval et ses langues (latine et vernaculaire). C'est ainsi que le deuxième temps, "Écrire », montre de quelle manière et à quelles fins Claudel intègre les éléments du monde médiéval et l'ancien français dans son œuvre. Le troisième et dernier temps, "Repenser », est construit en miroir: Claudel repense la Modernité à travers le Moyen Âge et le Moyen Âge à travers la Modernité - il l'adapte aux temps présents, le conçoit comme un vecteur d'expérimentation et l'inscrit dans le rêve scénique du Gesamtkunstwerk.

Ces éléments, qui donnent lieu à l'écriture d'un essai à paraître, doivent ainsi être pris comme une invitation à relire le médiévalisme claudélien, à accorder au Moyen Âge dans son œuvre un tout autre sens et une tout autre valeur, à en montrer l'imagination et la fantaisie.

\section{Architecture générale}

L'introduction s'applique dès lors à poser les fondements d'une compréhension globale de la question. La célébration claudélienne de l’homme médiéval, qui « apprend à regarder en contemplant et à composer en connaissant » (« Discours de réception à l'Académie Française, prononcé le 13 mars 1947 », CEuvres en prose, p. 643), est à double tranchant, dans les premières décennies du XXe siècle. Le

\footnotetext{
2 Parmi une vaste bibliographie, voir notamment Bassan 1995, Busser 1992, Madaule 1990, p. 167-188 et ReyFlaud 1973. Le Moyen Âge est également très présent dans des études portant sur des thèmes et des formes en particulier, les articles et ouvrages de Pascal Lécroart sont en particulier fondamentaux (Lécroart 1993, $2002,2004,2006$ ).
} 
contexte historique qui voit l'éclosion du corpus médiéval de Claudel s'avère défavorable et altère ainsi la valeur accordée au cadre médiéval de la pensée et de l'écriture du Poëte.

Dans la lignée de la bataille doctrinale du « Moyen Âge obscur » et du « Moyen Âge lumineux ", amorcée au XIX ${ }^{e}$ siècle, toute référence médiévale doit être lue à l'aune du concept, en soi mouvant, de modernité. Ne relèverait de la modernité que ce qui est inscrit dans un présent délesté de la doctrine chrétienne (fides), au profit de l'exaltation du seul matérialisme et de la rationalité (ratio). Le Moyen Âge chrétien de Claudel imposerait en ce sens une double force de résistance et ne pourrait $a$ priori prétendre au prestige exclusif de la modernité.

Le Moyen Âge claudélien est dès lors dominé par un malentendu initial, qu'un examen rigoureux suffit à dissiper. II n'est pas uniquement français, pas uniquement chrétien, mais accueille l'Orient et l'Occident, qui « existent à la fois dans le regard de Dieu » (Histoire de Tobie et de Sara, 2011, vol. 2, p. 708)³. II est marqué par une unité théologique, politique, temporelle et spatiale, de sorte qu'il devient I'unité de mesure d'autres temporalités. Le commentaire du verset, " Alors, ô Tyr, tu seras dans l'oubli soixante-dix ans comptés comme les jours d'un roi » (Isaïe, 23, 15), inverse ainsi l'ordre temporel biblique et la temporalité du monde sensible. Ces soixante-dix ans sont évoqués "comme s'il s'agissait d'un seul règne, d'un régime, d'une période d'un seul tenant, qui a son avènement, son apogée et son déclin, ainsi le Moyen Âge qui montre dans son développement une unité si remarquable » ("La Résurrection de Tyr », Supplément aux CEuvres Complètes, vol. 1, 1990, p. 175).

Le bénéfice que les études littéraires peuvent retirer des interactions et hybridations est alors bien supérieur à celui que procurent les actualisations du Moyen Âge. Ces interactions démultiplient le sens, entretiennent un dialogue entre des matières culturelles a priori incommensurables et s'inscrivent dans une esthétique foncièrement ouverte, à bien des égards moderne.

La pluralité des allusions, des structures et des thèmes empruntés au Moyen Âge soulève la question des sources, qui demeure en partie irréductible. Le Journal et le Catalogue de la Bibliothèque reflètent une partie des lectures de Claudel, sans toutefois permettre de déterminer avec certitude de ce qui a été lu partiellement, lu intégralement, lu dans le texte ou de seconde main. Le Littré semble lui avoir procuré nombre de citations, les Mémoires improvisés portant la trace de ses premiers contacts avec la littérature médiévale, quand M. Colin (1876-1878) lisait "La Chanson de Roland, Le Roman de Renart, enfin les textes qu'on ne lit pas d'habitude aux enfants » et qui I'« enthousiasmaient " (Mémoires improvisés, 1954, p. 19-20) ${ }^{4}$. Des représentations (de mystère pascal à Prague et du Mystère

\footnotetext{
${ }^{3}$ Sur cette coexistence, voir également l'évocation du Christ Pantocrator dans La Parabole du festin, dans " l'ouverture centrale de l'abside " se place " un Christ gigantesque en vêtements d'Or tenant le globe du monde dans sa main, tel qu'on le voit sur les mosaïques byzantines " (La Parabole du Festin, Théâtre, vo. 2, 2011, p. 549).

${ }^{4}$ Eugène Roberto note que " Hugo romancier et le Roman de Renart ont laissé des traces dans la farce et le drame féérique [L'Endormie] de Claudel » (Roberto 1963, p. 51).
} 
de la Passion à la Sorbonne) et des rencontres ont façonné une culture médiévale qui n'est en rien systématique ni dogmatique.

Si l'enseignement patristique et les écrits théologiques sont fondamentaux, à I'image de références à Guillaume de Saint-Thierry, Jean Gerson, Pierre d'Ailly et saint Bonaventure, le Moyen Âge claudélien n'exclut aucun domaine. Du traité d'arts libéraux (Noces de Mercure et de Philologie) à l'épopée (Girard de Roussillon, Raoul de Cambrai), de la lyrique occitane (Jaufré Rudel) à l'efflorescence allégorique du XIII siècle, son innutrition médiévale semble avoir suivi le précepte : omnia disce, videbis postea nichil esse superfluum (Saint-Victor, Didascalicon, VI, 3, 1991, p. 154) $)^{5}$.

La République chrétienne (respublica christiana), qui atteint au Moyen Âge son plus haut degré d'accomplissement, reste l'horizon de l'écriture claudélienne et non un paysage contemplé d'un promontoire des temps anciens. L'inspiration médiévale est à la fois remémoration et projection dans un perpétuel devenir, animé d'un esprit d'avant-garde. L'écriture et l'imaginaire médiévaux sont alternativement accordés et mis en tension avec les données d'autres siècles. Leur trajectoire suit un parcours parallèle à celui du médiévalisme, substituant à la seule fin esthétique et politique une fin homilétique, subordonnant les ressources de la modernité (cinéma, dramaturgie) à un questionnement plus profond encore.

L'innovatio prend, avec Claudel, un sens particulier. Elle ne relève plus d'une « conviction diffuse » selon laquelle « les problèmes spécifiques posés par la poésie et l'écriture médiévales rejoignent ce que l'on nomma naguère la modernité » (Zumthor 1980, p. 19), mais rassemble Moyen Âge et modernité au profit de la conviction absolue, inébranlable et puissante de la foi - dont l'exaltation constitue en soi un puissant vecteur d'expérimentation et de recherche.

Les trois parties qui composent cette étude, "Penser ", "Écrire » et "Repenser », tentent de présenter le lien de l'œuvre claudélienne au Moyen Âge, selon les modalités qui président à son activité d'écrivain. Le regard pénétrant sur le monde médiéval, regard de diplomate, de moraliste, est celui du Journal et de ses œuvres en prose. L'écriture, fondée sur l'intégration de cette pensée préalable, s'accomplit dans sa production poétique et dramatique, qui repense continuellement la nature et le sens de cette période. Ces trois verbes, dans leur nudité quelque peu abstraite, représentent trois modalités d'approche successives et unitaires.

Claudel construit par l'écriture un monde médiéval, auquel il emprunte figures, thèmes, formes et motifs, dans une langue elle-même héritière d'inflexions médiévales. Dans le médiévalisme, l'écriture achève le cercle de la pensée. Le médiévalisme claudélien, pour exalter la religion chrétienne, tend vers l'innovatio et accomplit le rêve de l'œuvre d'art totale. En ce sens, il parcourt de manière originale la ligne de crête qui divise et unit médiévité et modernité.

«Penser » le Moyen Âge revient à en penser le monde, c'est-à-dire à faire émerger une conception particulière de l'histoire, et sa langue, dans ses aspects

\footnotetext{
${ }^{5}$ Trad. Apprends tout, et tu verras ensuite que rien n'est superflu.
} 
morphologiques et philosophiques, dès lors qu'elle contient une représentation du monde. Cette pensée, pour reprendre des images claudéliennes, fermente et ensemence son écriture.

Le regard que pose Claudel sur le Moyen Âge est à double foyer. Extérieur, il tente d'en saisir et d'en ordonner les traces; intérieur, il présente des affinités avec les modes de pensée qui prévalaient alors. Trois traits, historique, linguistique et social, en constituent l'essence. Le medium aevum est à la fois inscrit dans l'histoire et envisagé comme un concept anhistorique, comme un pont reliant deux siècles, quelle que soit la temporalité considérée. Replacé dans la continuité de l'histoire, il est déterminé par deux coexistences, dont il se fait l'écho : celle d'un ordre savant et $d^{\prime}$ un ordre populaire; celle du latin et des langues vernaculaires. Cette vision d'ensemble des réalités médiévales invite à étudier ce qui unit fondamentalement Claudel et la pensée médiévale.

Il partage avec les médiévaux une perception symbolique du monde, une approche allégorique qui établit des correspondances entre le monde sensible et le monde divin. La Nature est un Livre dont le sens ne se révèle qu'à travers un déchiffrement, sur le modèle de l'exégèse biblique. Symboles et allégories fleurissent dans l'écriture claudélienne et colorent son univers de teintes comparables à celles du Roman de la Rose et du théâtre liturgique: I'évanescence de certaines figures théâtrales est le revers du degré d'exemplarité auquel elles atteignent. L'analogie des sensibilités médiévale et claudélienne est néanmoins une donnée toujours relative et nuancée : admiration, réserve et dérision règlent son rapport avec le monde médiéval.

Claudel ne considère pas la langue médiévale comme la rémanence d'une forme intermédiaire, mais semble en partager les fondements intellectuels. Comme pour chaque emprunt à la civilisation du Moyen Âge, le problème de la langue est intégré à une problématique plus vaste. Les enseignements médiévaux, en cette matière, croisent la pensée mallarméenne, recoupent les conclusions de Cratyle et rencontrent les signes graphiques de la tradition japonaise et chinoise.

Claudel reconstitue ce qui fait l'essence même de la langue médiévale, entendue non comme système morphologique et syntaxique, mais comme philosophie du langage. L'étymologie, la lettre et le nom en sont les principes. La pensée étymologique du poète, qui accorde à l'intuition une place que lui dénie l'approche philologique, évoque celle d'Isidore de Séville, partant celle des philosophes et des théologiens qui s'en font l'écho. L'étymologie de "cadavre ", décomposé en « ca(ro) da(ta) ver(mibus) » (chair abandonnée aux vers), en donne un aperçu.

Les vertus aléthiques et poétiques de ce procédé sont également contenues dans la lettre, qui devient un morphème, une unité sémantique à part entière - quand la linguistique ne la conçoit que comme un "graphème ». Claudel, à l'instar des médiévaux, accorde à chaque lettre un sens indépendant et en interaction : « lettre par lettre, ainsi on trouve tout dans un mot comme les instruments de la passion dans une tête de brochet!» («Idéogrammes occidentaux», CEuvres en prose, 
1965$, p. 82 $)^{6}$. Le nom propre, enfin, partage les prolongements sémantiques du nom commun. Claudel, dans de grandes scènes de Jeanne d'Arc au Bûcher et du Livre de Christophe Colomb, redonne vie à la croyance médiévale du nom (nomen) porteur d'un présage (omen).

Le monde médiéval reconstruit par Claudel présente la réitération de figures historiques et mythiques. Ces figures, empruntées majoritairement au domaine français, inscrivent dans son œuvre la rémanence d'une mémoire culturelle, qui s'exerce également dans la réminiscence de textes médiévaux, dont il semble s'approprier certaines composantes micro- et macrostructurales. Claudel recompose alors un monde médiéval dont il ne retient pas les syntagmes classiques - qui ont trait au monde chevaleresque -, privilégiant le monde de l'esprit.

L'écriture médiévale du poète constitue une réalité bien plus subtile et mouvante qu'elle ne le semble. Loin de pérenniser un langage immuable et d'imposer à contre-temps la rémanence d'une temporalité révolue, elle en repense la logique et soumet les formes médiévales à l'énonciation de sa propre voix. Claudel n'a pas les yeux rivés sur un passé lointain, mais ajuste continuellement l'articulation du Moyen Âge et de la modernité. De manière réversible, le Moyen Âge est repensé (notamment au théâtre) à partir des moyens intellectuels de la modernité, et la modernité à partir de ses racines médiévales - car, comme le rappelle Umberto Eco, « nous marchons et prions toujours dans les nefs des Cathédrales » (Eco 1995, p. 68$)^{7}$.

\section{Considérations conclusives}

La modernité médiévale perceptible dans le drame claudélien procède à la fois d'une approche réflexive livrant les éléments d'une pensée singulière, et d'une approche pragmatique intégrant dans son écriture des traits qu'il remodèle et incorpore en une unité organique nourrie d'influences variées. Le Moyen Âge se donne à la fois comme un point d'ancrage spirituel, artistique et littéraire, et comme un objet historique dont il s'applique à circonscrire le sens, par-delà ses multiples réalités. Ces deux approches, dominées par la contemplation et I'innutrition, s'enrichissent d'une troisième, qui invite à repenser réciproquement le Moyen Âge à travers la modernité et la modernité à travers le Moyen Âge. Ce miroir que se tendent l'une à l'autre ces deux périodes de l'histoire est à l'origine d'un phénomène subtil de perception indirecte.

Élargir ainsi le champ de la pensée historique inscrit de plein droit Claudel dans la sphère du médiévalisme et restitue la complexité d'un Moyen Âge qui ne se réduit pas à un âge d'or du christianisme. Claudel envisage l'« application de modèles médiévaux à des besoins contemporains »(Workman 1987, p. 32) ${ }^{8}$, ce dont son «Projet d'une église souterraine à Chicago »(1926) suffit à donner la mesure.

\footnotetext{
${ }^{6}$ Ces réflexions, inspirées par la lecture d'un ouvrage du P. Wieger, source d' « amusement », certes, mais aussi " inépuisable source d'intérêt ".

7 " We no longer dwell in the Parthenon, but we still walk and pray in the naves of the Cathedral ».

8 " [Medievalism is] the study of the Middle Ages, the application of medieval models to contemporary needs ».
} 
Inversement, il applique au modèle médiéval un regard contemporain. II instaure, partant, une logique du discours historique, conservant une acuité qui fait défaut à d'autres hommes de son siècle.

C'est pourquoi il éreinte, dans une page de son Journal, le pape Pie XI, qui au lieu de considérer l'articulation des deux mondes, les confond en un errement coupable :

Le pape s'élève avec véhémence contre l'art moderne et ne trouve rien de pire pour le blâmer que de le comparer aux productions du Moyen Âge ! C'est rassurant ! On ne voit pas que l'art de S.-Sulpice ait jamais été blâmé. Pourquoi les prêtres ont-ils si peu de goût ? (Journal, vol. 1, p. 1016 [octobre 1932])

S'il est déraisonnable de confondre critère historique et critère esthétique, il le serait tout autant de déceler un rapport de rivalité ou d'exacte identité, entre médiévité et modernité. L'une n'est pas la figure repoussoir de l'autre, mais en quelque sorte son révélateur, le truchement qui permet d'en mieux pénétrer le sens. Les structures de l'esprit médiéval, les nomenclatures qui ordonnent la représentation du monde, éclairent le monde moderne, qui a négligé et perdu les repères de la théologie. En miroir, les ressources esthétiques et techniques de la modernité transforment la scène médiévale en un espace d'expérimentation.

Repenser le monde moderne à travers le Moyen Âge instaure un dialogue avec une mémoire culturelle qui se donne comme une valeur de référence. Dans les contours d'un paysage comme dans le retour périodique des violences et des guerres, cette mémoire médiévale donne aux réalités du monde une vaste profondeur de champ. En dépit d'une apparente symétrie, repenser le Moyen Âge à travers la modernité se joue sur un autre plan, esthétique et dramatique.

Est moderne, dans cette acception, le degré de perfectionnement technique et formel auquel est parvenue l'époque à laquelle vit Claudel. Certaines formes anciennes étant frappées de caducité (cf. Neiva et Montandon 2014), il ne semble " pas 'difficile' de leur donner 'une forme moderne' » ("Une visite à Bâle », CEuvres en prose, 1965, p. 942), comme il le note à propos de la danse macabre. À la forme s'adjoignent des procédés - poétiques, scénographiques, cinématographiques -, dont l'application inscrit le drame médiéval à l'horizon de l'œuvre d'art totale (Gesamtkunstwerk).

En ce sens, le Moyen Âge n'est pas pour lui un pur objet historique, il n'est pas le reflet de la moindre mélancolie, mais il représente toujours un avenir. C'est pourquoi il convient de donner enfin à Claudel la place qui lui revient au sein du médiévalisme.

\section{Bibliographie}

BASSAN, Fernande. 1995. "Le cadre médiéval de L'Annonce faite à Marie. " Revue d'Histoire du Théâtre 185, 33-38.

BusSER, Bernard. 1992. "Paul Claudel et la Fête de l'Âne. "Bulletin de la Société Paul Claudel 127 (3), 19-29.

CHujo, Shinobu. 1990. "Jeanne d'Arc au bûcher entre le nô et la messe. » Kiyô, 40, 55-63. 
Claudel, Paul. 1965. "Discours de réception à l'Académie Française, prononcé le 13 mars 1947. » [1947]. Dans CEuvres en prose. Paris : Gallimard, 634-658.

CLAUDEL, Paul. 1965. Figures et paraboles [1936], « Une visite à Bâle. » Dans CEuvres en prose. Paris: Gallimard, 939-943.

Claudel, Paul. 1965. "Idéogrammes occidentaux. » [1926] Dans CEuvres en prose. Paris: Gallimard, 81-91.

Claudel, Paul. 1968. Journal, 2 vol. [1904-1932 et 1932-1955]. Paris: Gallimard.

Claudel, Paul. 1990. "La Résurrection de Tyr », Supplément aux CEuvres complètes, vol. 1, Lausanne : L'Âge d'Homme, 174-177.

ClaUdel, Paul. 2001. Mémoires improvisés. Quarante et un entretiens avec recueillis par Jean Amrouche [1954]. Paris : Gallimard.

CLAUDEL, Paul. 2011. Histoire de Tobie et de Sara [1942], Théâtre, vol. 2, 697743.

Claudel, Paul. 2011. L'Annonce faite à Marie [1948], Théâtre, vol. 2, Paris, Gallimard, «Pléiade », 769-847.

CORBELLARI, Alain. 2008. "Un problème de "littérature française générale". La lecture de la Bible en clé nationale. » Poétique 155, 283-294.

Eco, Umberto. 1995. Faith in Fakes. Travels in Hyperreality. Londres: Vintage.

GreGH, Fernand. 1955, "Paul Claudel. » Revue des deux mondes 8, 577-591.

Gally, Michèle. 2000. La Trace médiévale et les écrivains d'aujourd'hui. Paris : PUF.

GeNTRY, Francis G. et Ulrich Müller. 1991. «The Reception of the Middle Ages in Germany: An Overview. » Studies in Medievalism III (4), 399-422.

GREVIN, Benoît. 2015. "De l'usage du médiévalisme (et des études sur le médiévalisme...) » Ménestrel, 25 mars.

$<$ http://www.menestrel.fr/?De-l-usage-du-medievalisme-et-des-etudes-sur-lemedievalisme-en-Histoire\&lang=en $>$ (25.05.2021).

LECROART, Pascal. 1993. Jeanne d'Arc au bûcher de Paul Claudel et Arthur Honegger. Paris : Publimuses.

LECROART, Pascal. 2002. " La rencontre du langage dramatique et du langage musical dans Jeanne d'Arc au bûcher de Paul Claudel et Arthur Honegger. " Dans Le Dramatique et le Lyrique dans l'écriture poétique et théâtrale des XIXe et XXe siècles, dir. Alexandre-Bergues, Pascale \& Didier Alexandre, Besançon : Presses Universitaires de Franche-Comté, 175-189.

LECROART, Pascal. 2004. Paul Claudel et la rénovation du drame musical. Sprimont : Mardaga.

LECROART, Pascal. 2006. "Les sources de Jeanne d'Arc au bûcher d'après la bibliothèque de Paul Claudel. » Bulletin de la Société Paul Claudel 184 (4), 31-42.

MadAule, Jacques. 1950. Dire le Moyen Âge : hier et aujourd'hui. Université de Picardie, Paris : PUF.

NeIVA, Saulo \& Alain Montandon (éd.). Dictionnaire raisonné de la caducité des genres littéraires. Genève : Droz.

Poisson-GuefFIer, Jean-François. 2022. Paul Claudel et le Moyen Âge. Paris : Honoré Champion. [à paraître]

PoIzAT, Alfred. 1927. La poésie contemporaine de Mallarmé à M. Paul Valéry, Imprimerie de Monaco.

ReY-FLAUd, Henri. 1973. "Claudel et le théâtre médiéval. » Mélanges de langue et de littérature médiévales offerts à Pierre Le Gentil, professeur à la Sorbonne, par ses collègues, ses élèves et ses amis. Paris : SEDES, 715720.

ROBERTO, Eugène. 1963. L'Endormie de Paul Claudel ou la naissance du génie. Université d'Ottawa : Cahier Canadien Claudel 1. 
SAINT-VICTOR, Hugues de. [vers 1165] 1991. Didascalicon. L'art de lire, introduction, traduction et notes par Michel Lemoine. Paris : Cerf.

SARRAZAC, Jean-Pierre (éd.). 2010. Lexique du drame moderne et contemporain. Paris : Circé, Poche.

StAHULJAK, Zrinka. 2020. Médiéval contemporain. Pour une littérature connectée. Paris : Macula, "Anamnèses. Médiéval/Contemporain ».

WorKMAN, Leslie \& Heather Arden (éd.). 1987. « Medievalism in France 15001750. " Studies in Medievalism III/1. Cambridge: Boydell \& Brewer.

Zumthor, Paul. 1980. Parler du Moyen Âge. Paris : Les Éditions de Minuit. 


\title{
Résumé
}

Les détracteurs de Claudel n'ont cessé de le présenter comme un poète ancré dans le Moyen Âge chrétien. L'inspiration médiévale de Claudel, sensible de Tête d'Or au Chemin de la Croix $n^{\circ} 2$, dénierait ainsi à son art toute modernité. En considérant l'intégralité de son œuvre, il apparaît que le Moyen Âge n'implique en rien une régression. Le Moyen Âge claudélien est beaucoup plus vaste qu'on ne l'imagine : il n'est pas uniquement chrétien mais accueille toutes les traditions du monde. II ne fait pas seulement revivre les genres et formes de ce passé, mais les hybride. Le Moyen Âge est dès lors moins tourné vers le passé que vers l'avenir et semble, à sa manière, infiniment plus moderne que celui de ses contemporains. C'est ce que nous tentons de démontrer dans un essai à paraître chez Honoré Champion en mars 2022, Paul Claudel et le Moyen Âge.

\begin{abstract}
The ennemies of Claudel have always tried to depict him as a poet of the christian Middle Ages. In their opinion, his medieval inspiration, from Tête $d^{\prime}$ Or to Le Chemin de la Croix $n^{\circ} 2$, could not be modern. But if we consider his complete works, it seems that his vision of the Middle Ages is far more wide that we could think. This period is christian, but far more inclusive. Claudel resurrected many medieval forms and genres, but always mixed them with other literary traditions. Therefore, Middle Ages are not turned towards the past, but towards the future. Indeed, Claudel is far more modern that his contemporaries. That is what we intend to demonstrate in a new essay called Paul Claudel and the Middle Ages, to be published by Champion in March 2022.
\end{abstract}

